\title{
WEAKLY UNIQUE FACTORIZATION MODULES
}

\author{
KÜRŞAT HAKAN ORAL, ÜNSAL TEKIR AND AHMET GÖKSEL AĞARGÜN
}

\begin{abstract}
In this work we give the definition of weakly prime element of a module. Therefore we give a new definition of factorization in a module, which is called weakly factorization. So we call a module weakly unique factorization module if all elements have a weakly factorization which is unique. We give the relation between weakly prime elements and weakly prime submodules. Then we characterize such weakly unique factorization modules.
\end{abstract}

The study of factorization in torsion-free modules was begun in Nicolas [5]. She defined the module $M$ to be factorial if (1) every nonzero element of $M$ has a irreducible factorization, (2) every irreducible element of $R$ is prime, and (3) every irreducible element of $M$ is primitive. She showed that if $M$ is factorial then $R$ is a UFD. After this she showed that $M$ is a unique factorization module (UFM) if and only if (1) every element of $M$ has an irreducible factorization, and (2) if $x=a_{1} a_{2} \cdots a_{k} m=b_{1} b_{2} \cdots b_{t} m^{\prime}$ are two factorizations of $x \in M$ then $k=t, a_{i} \sim b_{i}$ for all $i \in\{1,2, \ldots, k\}$ and $m \sim m^{\prime}$. Later, $\mathrm{Lu}$ [3] gives some characterizations of UFM and relations between prime submodules and primitive elements such modules. Further she investigates polynomial modules. There is an another work about factorizations of modules, by Anderson and Valdes-Leon [1]. They generalize factorization of any modules over a ring with zero divisor, which have nonzero torsion elements. They showed that their definition and definition of Nicolas are concides if $M$ is torsion-free module and $R$ is an integral domain.

We give a new definition of factorization for modules, named weakly factorization, and give relations between weakly prime elements and weakly prime submodules. After this we investigate the direct sum of modules, the direct product of modules, fractions of modules and polynomial of modules.

Throughout this paper all rings, $R$ are commutative ring with identity 1 and all modules, $M$ are nonzero torsion-free $R$-module which are unitary. An $R$-module $M$ is called a torsion-free module if $r m=0$ for some nonzero $r \in R$ and $m \in M$ then $m=0$. Let $R$ be a ring, $M$ a nonzero torsion free $R$-module and $U(R)$ the set of all unit elements of $R$. Let $m$ and $m^{\prime}$ be two elements of $M$. We say that $m$ divides $m^{\prime}$ in $M$, written $m \mid m^{\prime}$, if there exists a nonzero element $r \in R$ such that $m^{\prime}=r m$. If $m \mid m^{\prime}$, then $m$ is called a factor or divisor of $m^{\prime}$ in $M$. Similarly, an element $d \in R$ is said to divide $m$

Corresponding author: Kürşat Hakan Oral.

Received May 21, 2009.

Key words and phrases. Weakly prime element, weakly prime submodule, factorization. 
in $M$, written $d \mid m$, if there exists an element $m_{0} \in M$ such that $m=d m_{0}$. If $m \mid m^{\prime}$ and $m^{\prime} \mid m$ then we shall say that these elements are associates in $M$ and write $m \sim m^{\prime}$. Clearly, $m \sim m^{\prime}$ if and only if $m=u m^{\prime}$ and $v m=m^{\prime}$ for some $u, v \in U(R)$. If $m \mid m^{\prime}$ and $m$ is not an associate of $m^{\prime}$, then we say that $m$ is a proper factor of $m^{\prime}$ in $M$.

We will give now some definitions from [3] and [4].

Definition 1. Let $M$ be a torsion-free $R$-module and $m$ be a nonzero element of $M$.

(i) $m$ is irreducible in $M$ if $m=a m^{\prime}$ implies that $a \in U(R)$ for every $a \in R$ and $m^{\prime} \in M$.

(ii) $m$ is primitive in $M$ if $m \mid a m^{\prime}$ implies $m \mid m^{\prime}$ for all $0 \neq a \in R$ and $m^{\prime} \in M$.

(iii) An irreducible element $p$ of $R$ is called prime to the module $M$ if $p \mid a m$ implies $p \mid a$ in $R$ or $p \mid m$ in $M$.

Definition 2. Let $M$ be an $R$-module. Then a submodule $N$ of $M$ is called pure submodule if for all $a \in R$ we have $a M \cap N=a N$.

The following Propositions are given by [3] without their proofs, we will give now their proofs.

Proposition 1. Let $M$ be an $R$-module then every $w$-primitive element of $M$ is an irreducible element.

Proof. Suppose that $m$ is a primitive element of $M$ and let $m=a m^{\prime}$ for some $a \in R$, $m^{\prime} \in M$. Then $m \mid a m^{\prime}$ and since $m$ is primitive we get $m \mid m^{\prime}$. Since $m=a m^{\prime}$ implies $m^{\prime} \mid m$. Therefore $m \sim m^{\prime}$, hence $m$ is a irreducible element.

Proposition 2.[3, Proposition 1.1] Let $M$ be an R-module and $0 \neq m \in M$, then the following statements are equivalent:

(i) $m$ is a primitive element of $M$,

(ii) the cyclic submodule $R m$ is a pure submodule of $M$ and

(iii) for every $x \in M$ we have either $R x \cap R m=0$ or $R x \subseteq R m$.

Proof. $(i) \Rightarrow($ ii $)$ Let $m$ be a primitive element of $M$ and $a \in R$. Now suppose that $x \in a M \cap R m$, then there exist elements $m^{\prime} \in M$ and $r \in R$ such that $x=a m^{\prime}=r m$. Thus $m \mid a m^{\prime}$ and since $m$ is primitive, we have $m \mid m^{\prime}$. Therefore there exist $r^{\prime} \in R$ such that $m^{\prime}=r^{\prime} m$. So $x=a m^{\prime}=a r^{\prime} m \in a R m$. For the converse, let $y \in a R m$ then there exists an $r \in R$ such that $y=a r m$. So it is clear that $y \in a M$ and $y \in R m$.

$($ ii $) \Rightarrow($ iii $)$ Suppose that $R m$ is pure submodule of $M$ and let $x \in M$ such that $R x \cap R m \neq$ 0 . Then there exists $y \in R x \cap R m$ such that $y=r^{\prime} x$ for some $r^{\prime} \in R$. Since $R m$ is pure submodule of $M$ we get $r^{\prime} M \cap R m=r^{\prime} R m$. So $y=r^{\prime} x \in r^{\prime} M \cap R m=r^{\prime} R m$. Thus there exist $r^{*} \in R$ such that $y=r^{\prime} x=r^{\prime} r^{*} m$. Hence $x=r^{*} m$ and this gives $R x \subseteq R m$. (iii) $\Rightarrow(i)$ Suppose that for all $x \in M$ either $R x \cap R m=0$ or $R x \subseteq R m$. Now assume that $m \mid a m^{\prime}$ for some $a \in R$, then there exists an $r \in R$ such that $a m^{\prime}=r m$. Thus $R m^{\prime} \cap R m \neq 0$ and so by our assumption we get $R m^{\prime} \subseteq R m$. Then there exists a $s \in R$ such that $m^{\prime}=s m$ and this gives $m \mid m^{\prime}$. So $m$ is primitive. 
Corollary 1.([3, Corollary] $]$ Let $M$ be an $R$-module then two primitive elements $m$ and $m^{\prime}$ of $M$ are non-associates if and only if $R m^{\prime} \cap R m=0$.

Definition 3. A nonzero element $m$ of $M$ is called weakly prime ( $w$-prime) if for $a, b \in R$ and $m^{\prime} \in M, m \mid a b m^{\prime}$ implies $m \mid a m^{\prime}$ or $m \mid b m^{\prime}$.

Note that if $m$ is a $w$-prime element of $M$ then $r m$ is $w$-prime too for all $r \in U(R)$.

Proposition 3. Let $M$ be an $R$-module, then every primitive element of $M$ is w-prime.

Proof. Assume that $m \mid a b m^{\prime}$ for some $a, b \in R$ and $m^{\prime} \in M$. Then since $m$ is primitive, we get $m \mid m^{\prime}$. Hence $m \mid a m^{\prime}$ and $m \mid b m^{\prime}$.

Example 1. Let $R$ be a commutative ring with an identity and $M=R[x]$, the polynomial ring over $R$ is an $R$-module, then $x \in M$ is w-prime (primitive, irreducible) element.

Example 2. Let $R=\mathbb{Z}$ and $M=\mathbb{Z}[x]$. Then the element $2 x$ is a w-prime element but is neither primitive nor irreducible.

Theorem 1. Let $M$ be a cyclic module Rx over a UFD R and $m \in M$ such that $m \nsim x$. Then the following statements are equivalent;

(i) $m \in M$ is a w-prime element,

(ii) $m \sim p x$ for some irreducible element $p$ of $R$,

(iii) $R m=p M$ for some irreducible element $p$ of $R$.

Proof. $(i) \Rightarrow(i i)$ Let $m$ be a $w$-prime element of $M$. Here $m \in M=R x$ and there exists an element $r \in R$ such that $m=r x$. Since $R$ is UFD $r$ has a factorization such that $r=p_{1} \cdots p_{k}$ where $p_{i} \in R$ are all irreducible. Since $m$ is a $w$-prime element of $M$, $m \mid p_{i} x$ for some $i \in\{1,2, \ldots, k\}$. Hence $m \sim p_{i} x$.

(ii) $\Rightarrow($ iii $)$ Let $m \sim p x$ for some irreducible element $p$ of $R$. Then there exists a unit element $u \in R$ such that $m=u p x=p u x \in p R x=p M$. If $p n \in p M$ where $n \in M$ then for some $s \in R, p n=p s x=s p x=s u^{-1} m \in R m$. Thus $R m=p M$.

$($ iii $) \Rightarrow($ i) Let $R m=p M$ for some irreducible element $p$ of $R$. Assume that $m \mid a b n$ for some $n \in M$ and $a, b \in R$. Then there exists an $r \in R$ such that $r m=a b n$. Since $R m=p M$ and $M=R x$ we get for some $m^{\prime} \in M$ and $r^{\prime}, r^{\prime \prime} \in R$, abn $=a b r^{\prime} x$ and $r m=p m^{\prime}=p r^{\prime \prime} x$. Thus $a b r^{\prime}=p r^{\prime \prime}$ and $p \mid a b r^{\prime}$. Therefore we obtain $p \mid a$ or $p \mid b$ or $p \mid r^{\prime \prime}$. If $p \mid a$ we get $m \mid a n$ and if $p \mid b$ we get $m \mid b n$. Therefore we have $m \mid n$.

Definition 4. A submodule $N$ of an $R$-module $M$ is called weakly prime if $a b k \in N$ implies $a k \in N$ or $b k \in N$ for all $k \in M$ and $a, b \in R$.

Theorem 2. A submodule $N$ of an $R$-module $M$ is called weakly prime if and only if $a b K \subseteq N$ implies $a K \subseteq N$ or $b K \subseteq N$ for some submodule $K$ of $M$ and $a, b \in R$. 
Proof. Suppose that $N$ is a weakly prime submodule of $M$. Let $a b K \subseteq N$ for some submodule $K$ of $M$ and $a, b \in R$. Since for all $k \in K$ we have $a b k \in N$ we get $a k \in N$ or $b k \in N$. Hence $a K \subseteq N$ or $b K \subseteq N$. Indeed, if there are not $k_{1}, k_{2} \in K$ such that $a k_{1} \in N$ but $b k_{1} \notin N$ and $b k_{2} \in N$ but $a k_{2} \notin N$, then we let the element $k_{1}+k_{2} \in K$ we obtain $a b\left(k_{1}+k_{2}\right) \in N$. Thus $a\left(k_{1}+k_{2}\right) \in N$ or $b\left(k_{1}+k_{2}\right) \in N$. If $a\left(k_{1}+k_{2}\right) \in N$ then $a k_{2} \in N$ which is a contradiction. If $b\left(k_{1}+k_{2}\right) \in N$ then $b k_{1} \in N$ which is a contradiction. For the converse let $a b k \in N$ for some $k \in M$. Then $a b(k) \subseteq N$. By our assumption $a(k) \subseteq N$ or $b(k) \subseteq N$. Thus $a k \in N$ or $b k \in N$.

Theorem 3. Let $M$ be an $R$-module. Then $m \in M$ is w-prime element if and only if $R m$ is w-prime submodule of $M$.

Proof. Let $m$ be a $w$-prime element. Assume that for any $k \in M$ and $a, b \in R$ we have $a b k \in R m$, thus $m \mid a b k$. Since $m$ is $w$-prime in $M, m \mid a k$ or $m \mid b k$. This implies $a k \in R m$ or $b k \in R m$. Now for the converse suppose that $R m$ is a $w$-prime submodule of $M$. Assume that $m \mid a b m^{\prime}$ for some $a, b \in R$ and $m^{\prime} \in M$. Thus $a b m^{\prime} \in R m$ and since $R m$ is $w$-prime submodule, we get $a m^{\prime} \in R m$ or $b m^{\prime} \in R m$. Hence $m \mid a m^{\prime}$ or $m \mid b m^{\prime}$.

Here first we shall note that if we let $R$ as a module over itself then an element $m$ is $w$-prime of the module $R$ if and only if $m$ is a prime element of the ring $R$. Secondly every primitive element of $M$ is $w$-prime.

Definition 5. A torsion-free module $M$ over a commutative ring with identity $R$ is called a weakly unique factorization module ( $w$-UFM) or $w$-factorial module if the following two conditions are satisfied:

(w-ufm 1) Each nonzero element $x \in M$ has a $w$-factorization, $x=a_{1} a_{2} \cdots a_{k} m$, where $a_{i}$ 's are ireducible elements in $R$ (possibly with $k=0$ ) and $m$ is a $w$-prime element in $M$.

(w-ufm 2) if $x=a_{1} a_{2} \cdots a_{k} m=b_{1} b_{2} \cdots b_{t} m^{\prime}$ are two factorizations of $x$, then $k=t$, $a_{i} \sim b_{i}$ and $m \sim m^{\prime}$ for all $i \in\{1,2, \ldots, k\}$.

Definition 6. Let $M$ be an $R$-module and $a \in R, m \in M$

(1) An element $d \in R$ is called greatest common divisor ( $\mathrm{gcd}$ ) of $a$ and $m$ if the following two conditions hold

(i) $d \mid a$ in $R$ and $d \mid m$ in $M$, and

(ii) if there is an element $c \in R$ such that $c \mid a$ in $R$ and $c \mid m$ in $M$ then $c$ is a divisor of $d$.

(2) An element $m^{\prime} \in M$ is called least common multiple (lcm) of $a b$ and $m$ if the following two conditions hold

(i) $a \mid m^{\prime}$ and $m \mid m^{\prime}$ in $M$ respectively, and

(ii) if there is an element $n \in M$ such that $a \mid n$ and $m \mid n$ in $M$ then $m^{\prime}$ is a factor of $n$. 
Proposition 4.([3, Proposition 1.2]) Let $M$ be an $R$-module then

(i) $m^{*} \sim \operatorname{lcm}\{a, m\}$ if and only if $a M \cap R m=R m^{*}$.

(ii) Let $p$ be an irreducible element of $R$ such that $\operatorname{lcm}\{p, a m\}$ exists in $M$ for any $a \in R$. If $p \nmid a m$ then $p M \cap R a m=R p a m$.

Proposition 5.([3, Proposition 1.3]) Let $M$ be an $R$-module and $R$ a $G C D$ domain then

(1) $\operatorname{gcd}\{(a, c), m\}=\operatorname{gcd}\{a, \operatorname{gcd}\{c, m\}\}$

(2) $\operatorname{gcd}\{c a, c m\}=c \operatorname{gcd}\{a, m\}$ and

(3) if $a \mid c m$ and $\operatorname{gcd}\{a, m\}=1$ then $a \mid c$.

Theorem 4. Let $M$ be a module over a UFD $R$ which satisfies (w-ufm 1$)$. Then $M$ is a $w$-UFM if and only if every weakly prime element of $M$ is primitive.

Proof. Let $M$ be a $w$-UFM and $m \in M$ be a weakly prime element. Assume that $m \mid a b m^{\prime}$ for some $m^{\prime} \in M$ and $a, b \in R$. Then there exist an $r \in R$ such that $r m=a b m^{\prime}$. Since $M$ is a $w$-UFM and $R$ is a UFD there exist irreducible elements in $R$, $r_{1}, \ldots, r_{k}, a_{1}, \ldots, a_{t}, b_{1}, \ldots, b_{l}, c_{1}, \ldots, c_{n}$, and weakly prime element $m^{*}$ in $M$ such that $r=r_{1} \cdots r_{k}, a=a_{1} \cdots a_{t}, b=b_{1} \cdots b_{l}$ and $m^{\prime}=c_{1} \cdots c_{n} m^{*}$. Thus we get $r_{1} \cdots r_{k} m=$ $a_{1} \cdots a_{t} b_{1} \cdots b_{l} c_{1} \cdots c_{n} m^{*}$. Since $M$ is a $w$-UFM we have by the uniqueness, $k=t+l+n$ and for a suitable choice $r_{i} \sim a_{i}, r_{j} \sim b_{j}, r_{s} \sim c_{s}$ and $m \sim m^{*}$. Therefore there exist an $r^{*} \in U(R)$ such that $m^{*}=r^{*} m$. Hence $m^{\prime}=c_{1} \cdots c_{n} m^{*}=c_{1} \cdots c_{n} r^{*} m$. Thus $m \mid m^{\prime}$ and so $m\left|a m^{\prime}, m\right| b m^{\prime}$. Now suppose that every weakly prime element of $M$ is primitive and $x=a_{1} \cdots a_{t} m=b_{1} \cdots b_{l} m^{\prime}$ are two decompositions of $x$. Then we have $m\left|b_{1} \cdots b_{l} m^{\prime}, m^{\prime}\right| a_{1} \cdots a_{t} m$. Since $m$ and $m^{\prime}$ are $w$-prime elements this gives us $m \mid m^{\prime}$ and $m^{\prime} \mid m$. Hence $m \sim m^{\prime}$, so there exist an elemnet $u \in U(R)$ such that $m=u m^{\prime}$. Therefore, $a_{1} \cdots a_{t} u m^{\prime}=b_{1} \cdots b_{l} m^{\prime}$ implies $u a_{1} \cdots a_{t}=b_{1} \cdots b_{l}$. The result follows since $R$ is a UFD.

Theorem 5. Let $M$ be a torsion-free $R$-module. Then $M$ is a UFM if and only if $M$ is $w-U F M$.

Proof. The follows from Theorem 4 and [3, Theorem 2.1].

With this theorem we get that in a $w$-UFD $M$, every weakly prime element of $M$ is irreducible element of $M$. And this gives us that weakly factorial modules and factorial modules concides. From this note we obtain the following corollaries.

Corollary 2. Every vector space is w-UFM.

Theorem 6. Let $M$ be a module over a UFD $R$ which satisfies (w-ufm 1). Then the following statement are equivalent:

(i) Every weakly prime element of $M$ is weakly primitive 
(ii) For any pair of elements $a, b \in R$ and $m \in M$ the gcd $\{a b, m\}$ exists in $R$.

(iii) For any pair of elements $a, b \in R$ and $m \in M$ the $l c m\{a b, m\}$ exists in $M$, i.e. the submodule $a M \cap R m$ is cyclic.

(iv) Every irreducible element $p$ of $R$ is prime to $M$.

Note that if we let $S$ be a multiplicative closed subset of a UFD $R$ such that $0 \notin S$ then by Theorem 5 and [3, Proposition 5.1] we obtain that if $M$ is a $w$-factorization $R$-module then the module of fractions $M_{s}$ is a $w$-factorization $R_{s}$-module. For the converse, let $S$ be a the multiplicative closed subset of $R$ generated by any family $P^{\prime}$ of elements which are $w$-prime to $M$. If $M_{s}$ is a $w$-factorization $R_{s}$-module, then $M$ is a $w$-factorization $R$-module in the sense of Theorem 5 and [3, Proposition 5.2].

Similarly, by Theorem 5 and [3, Proposition 6.1] and [3, Theorem 6.1] we get $w$ factorization of $M[x]$, polynomial module over $M$, is a $w$-factorization module over the polynomial ring over $R, R[x]$.

Theorem 7. Let $M$ be a $w$-factorization module over a UFD $R$ such that $p M \neq M$ for every non-unit element $p \in R$. Then the following statements are equivalent:

(i) $p$ is prime to $M$,

(ii) $p M$ is a weakly prime submodule of $M$ with $(p M: M)=(p)$.

Proof. $(i) \Rightarrow($ ii $)$ Let $p \in R$ be a $w$-prime element to $M$ and let $a b k \in p M$ for some $a, b \in R$ and $k \in M$. Since $p$ is weakly prime to $M$ and $R$ is UFD $p \mid a b k$ implies that $p \mid a$ or $p \mid b$ or $p \mid k$. Assume that $b k \notin p M$ then since $p$ is prime to $M$ and $R$ is UFD, $p \mid a$. Thus $p \mid a k$. So $p M$ is a weakly prime submodule of $M$. For the second part $(p M: M) \supseteq(p)$ is clear, now let $r \in(p M: M)$. Then for $m \in M \backslash p M$ we have $r m \in p M$. Thus $p \mid r m$. Since $p$ is prime to $M$ we get $p \mid r$. Hence $r \in(p)$.

$(i i) \Rightarrow(i)$ Suppose that $p M$ is a weakly prime submodule of $M$ with $(p M: M)=(p)$. First we show that $p$ is an irreducible element of $R$. Let $p=a b$ for some $a, b \in R$, then since $p M$ is $w$-prime submodule $a b M \subseteq p M$ implies that $a M \subseteq p M$ or $b M \subseteq p M$. So $a \in(p M: M)=(p)$ or $b \in(p M: M)=(p)$. Thus $b$ is a unit or $a$ is a unit. Now secondly let $p \mid a b m$ but $p \nmid a b$ for some $a, b \in R$ and $m \in M$. Thus $p \mid a m$ or $p \mid b m$. If $p \mid a m$ then there exists an element $m^{*} \in M$ such that $p m^{*}=a m$. Since $M$ is $w$-factorization and $p \nmid a$ we get $p \mid m$.

Theorem 8. Let $\left\{M_{i} \mid i \in I\right\}$ be a set of modules over a UFD R. Then the following statements are equivalent:

(i) $\prod_{i \in I} M_{i}$ is a $w$-UFM over $R$,

(ii) $\bigoplus_{i \in I} M_{i}$ is a w-UFM over $R$,

(iii) each $M_{i}$ is a $w$-UFM over $R$. 
Proof. $(i) \Rightarrow(i i) \Rightarrow($ iii $)$ it's clear.

(iii) $\Rightarrow(i)$ Now assume that each $M_{i}$ is a $w$-UFM over $R$ for $i \in I$. Let $M=\prod_{i \in I} M_{i}$ and $m=\left(m_{i}\right)_{i \in I} \in M$ where $m_{i}=a_{i} m_{i}^{\prime}$ for some $a_{i} \in R$ and a $w$-prime element $m_{i}^{\prime}$ of $M_{i}$. First we will show that $m=\left(m_{i}\right)_{i \in I} \in M$ is a $w$-prime element in $M$ iff $\left\{a_{i}\right\}_{i \in I}$ has no g.c.d. in $R$. Let $m \in M$ be a $w$-prime element. Assume that $d=$ g.c.d. $\left\{a_{i}\right\}$ and set $a_{i}=d b_{i}$ for $b_{i} \in R$. Then $m=d m^{\prime}$ where $m^{\prime}=\left(b_{i} m_{i}^{\prime}\right)_{i \in I}$. Then $m \mid m=d m^{\prime}$ but $m \nmid m^{\prime}$ gives us a contradiction. For the converse assume that $\left\{a_{i}\right\}_{i \in I}$ has no g.c.d. in $R$. Let $m \mid c b n$ for some $c, b \in R$ and $n=\left(n_{i}\right)_{i \in I} \in M$ then there exist $r \in R$ such that $r m=c b n$. So this gives us that for all $i \in I, r m_{i}=c b n_{i}$. Thus for all $i \in I$, $r a_{i} m_{i}^{\prime}=c b n_{i}$. Since $m_{i}^{\prime}$ is $w$-prime and $\left\{a_{i}\right\}_{i \in I}$ has no g.c.d. then for all $i \in I$ we get $a_{i} m_{i}^{\prime} \mid c n_{i}$ or $a_{i} m_{i}^{\prime} \mid b n_{i}$. Hence $m \mid c n$ or $m \mid b n$, so $m$ is $w$-prime. Now we will show that $M$ is a $w$-UFM. Let $m=\left(m_{i}\right)_{i \in I} \in M$, since each $M_{i}$ is a $w$-UFM over $R$ we have a $w$-factorization for $m_{i} \in M_{i}$ and $i \in I$ such that $m_{i}=a_{i} m_{i}^{\prime}$ where $m_{i}^{\prime}$ is $w$-prime in $M_{i}$. If we let $d=$ g.c.d. $\left\{a_{i}\right\}$ then for $a_{i}=d b_{i}$ we obtain the equition $m=d m^{\prime}$ where $m^{\prime}=\left(b_{i} m_{i}^{\prime}\right)$. Now by Theorem $7 \mathrm{~m}^{\prime}$ is $w$-prime in $M$ and since $R$ is UFD $M$ satisfies $w$-UFM1. Now, let $p \in R$ be a $w$-irreducible element such that $p \mid a b m$ in $M$ for some $a, b \in R$ and $m=\left(m_{i}\right)_{i \in I} \in M$. Then for all $i \in I, p \mid a b m_{i}$ in $M_{i}$. Since $M_{i}$ is $w$-UFM if $p \nmid a b$ then $p \mid m_{i}$ for all $i \in I$. Consequently $p \mid m$ and therefore $M$ is $w$-UFM.

Corollary 3. Every free module over a UFD is w-UFM.

Corollary 4.([3, Theorem 5.1]) Let $S$ be a multiplication closed set of aUFD $R$ such that $0 \notin S$. If $M$ is a w-factorization $R$-module, then the module of fractions $M_{S}$ is a $w$-factorization $R_{S}$-module.

Corollary 5.([3, Theorem 5.2]) Let $M$ be a module over a UFD $R$ which satisfies (w-ufm $1)$ and $S$ be the multiplicatively closed set of $R$ generated by any family $P^{\prime}$ of elements which are w-prime to $M$. If the $R_{S}$-module $M_{S}$ is $w$-factorization so is the $R$-module $M$.

Corollary 6. ([3, Proposition 6.1]) Let $R$ be an integral domain satisfying the a.c.c. for principal ideals and $M$ an $R$-module. If $M$ satisfies the a.c.c. for cyclic submodules, so do the $R[x]$-module $M[x]$.

Corollary 7. ([3, Theorem 6.1]) If $M$ is a w-factorization module over a UFD $R$, then the $R[x]$-module $M[x]$ is also $w$-factorization.

\section{References}

[1] D. D. Anderson and S. Valdes-Leon, Factorization in Commutative Rings with Zero Divisors II, Factorization in integral domains (Iowa City, IA, 1996), 197-219, Lecture Notes in Pure and Appl. Math., 189, Dekker, New York, 1997.

[2] A. Azizi, Weakly prime submodules and prime submodules, Glasgow Math. J., 48(2006), 343-346. 
[3] Chin-Pi Lu, Factorial modules, Rocky Mountain J. Math., 7 (1977), 125-139.

[4] A. Nicolas, Modules Factoriels, Seminaire Dubrell-Pisot, 1966/67, no.10

[5] D. G. Northcott, Lesson on Rings,Modules and Multiplicities, Cambridge University Press, 1968

Yıldız Technical University, Department of Mathematics, 34720, Istanbul, Turkey.

E-mail: khoral@yildiz.edu.tr

Marmara University, Department of mathematics, Ziverbey, Goztepe,34722, Istanbul, Turkey.

E-mail: utekir@marmara.edu.tr

Yıldız Technical University, Department of Mathematics, 34720, Istanbul, Turkey.

E-mail: agar@yildiz.edu.tr 